\title{
Design of an Automatic Windshield-Breaking Device for Abnormal Situations in Moving Vehicles
}

Ching-Sung Wang ${ }^{1 *}$, Teng-Wei Wang ${ }^{2}$ and Min-Chun Zhou ${ }^{1}$

${ }^{1}$ Department of Electronic Engineering, Oriental Institute of Technology, New Taipei 220, Taiwan

${ }^{2}$ Department of Thoracic, Zhangzhou City Third Hospital, Fujian, China

\begin{abstract}
In recent years, cases of vehicles being accidentally driven into the sea or flooded sewers have been frequently reported. However, due to strong external water pressure in such situations, drivers sometimes fail to open a door for escape, causing irreversible results. Moreover, the inhalation of dangerous gases, as generated by the air conditioner and circulated in the vehicle, can cause permanent damage to the occupants of the vehicles. Considering the abovementioned scenario, this author believes that if a device can break the windshield and automatically make an emergency phone call when the vehicle is under water and there is a gas leakage, it would enable drivers and passengers to immediately escape, thus, effectively improving their safety. Therefore, this study focuses on this issue as its major research direction and aim; moreover, a review of domestic and overseas research regarding vehicle safety devices and accessory products shows that such windshield-breaking devices do not exist.
\end{abstract}

Keywords: Automatic windshield breaking device; Abnormal situation; Vehicle; Water detection; Abnormal gas detection

\section{System Design}

This study focuses on the collocation and application of sensors and chips, which feature easy and stable operation. After determining all hardware structures, the circuits could be designed using the Arduino Nano plate as its core, and through intense integration testing, avoid errors or problems [1]. Hence, the implementation of each procedure requires multiple testing times, in order that the most effective plans can be selected. The design procedure of the system is showed in Figure 1.

\section{Hardware Structure}

The entire circuit design plan is shown in Figure 2. The major power supply is the car battery, which drives the electromagnet and voltage-stabilizing circuit to drive the chip and peripheral circuits, thus, realizing the automatic braking and emergency call functions.

\section{Electromagnet-controlled circuit}

The control chip sends a signal, either high or low, to control the transistor in the saturation region and cutoff region. When the control signal is high, the saturation region of the transistor short circuits, which connects the coil, followed by the NO pin-out and COM pinout of the relay (943-1C-5DS) to enable the electromagnet to run. When the control signal is low, the cutoff region of the transistor is an open circuit, meaning the NC pin-out and COM pin-out of the relay reconnect, which is the same as at the initial state. The circuit is shown in Figure 3. Additionally, the supply voltage of the electromagnet is a $12 \mathrm{~V}$-supply for vehicles, while the chip and the peripheral circuit can be run by a $5 \mathrm{~V}$-supply. To avoid the voltage from being too high, the IC 7805 is used to stabilize the voltage. In tests, when the input voltage is higher than $5 \mathrm{~V}$, the stabilization system is effective [2].

This system uses dc electromagnets, and the magnetic field generated from the direct current passing through the coil causes the iron core to bounce. The strength of the bounce differs as the coil turns, thus, the magnitude of the current and the material of the iron core change, and due to the inertia effect, the strength of the impact force can be effective [3]. The dc electromagnet system is showed in Figure 4.

\section{Water level circuit}

This circuit uses the switch circuit by integrating the relay (LU-5H) and transistor (2SD313). The saturation region and cutoff region of the transistor are mainly used. When Wire 1 and Wire 2 are connected, the transistor is in its saturation region and short circuits, which enable the coil of the relay, the NO pin-out, and COM-out to connect and transmit a low signal. When the wires are not connected, the transistor is in its cut off region, the relay does not change, and the NC pin-out remains connected with the COM pin-out to transmit high signals [4]. By using such high and low signals, the chips can design their actions. The detecting circuit is showed in Figure 5.

\section{MQ-7 module}

The MQ series has a module to detect multiple gases, and MQ-7 is used for the core of this study to detect carbon monoxide. The module has both analog and digital outputs, and the concentration rises linearly as the voltage rises, thus, qualifying itself as proof to determine the concentration [5].

\section{Bluetooth module}

The Bluetooth device used in this study has principal and subordinate integration, which can actively search for mobile phones to connect and be passively searched [6,7]. With this module, the connection is possible simply by planning digital pin-outs for RX and $\mathrm{TX}$, and the Bluetooth device has low power consumption.

*Corresponding author: Ching-Sung Wang, Department of Electronic Engineering, Oriental Institute of Technology, New Taipei 220, Taiwan, Tel: 886921207288; Fax: 886-2-77385456; E-mail: ff020@mail.oit.edu.tw

Received November 21, 2017; Accepted December 11, 2017; Published December 17, 2017

Citation: Wang CS, Wang TW, Zhou MC (2017) Design of an Automatic Windshield-Breaking Device for Abnormal Situations in Moving Vehicles. J Electr Electron Syst 6: 247. doi: 10.4172/2332-0796.1000247

Copyright: ( 2017 Wang CS, et al. This is an open-access article distributed under the terms of the Creative Commons Attribution License, which permits unrestricted use, distribution, and reproduction in any medium, provided the original author and source are credited. 


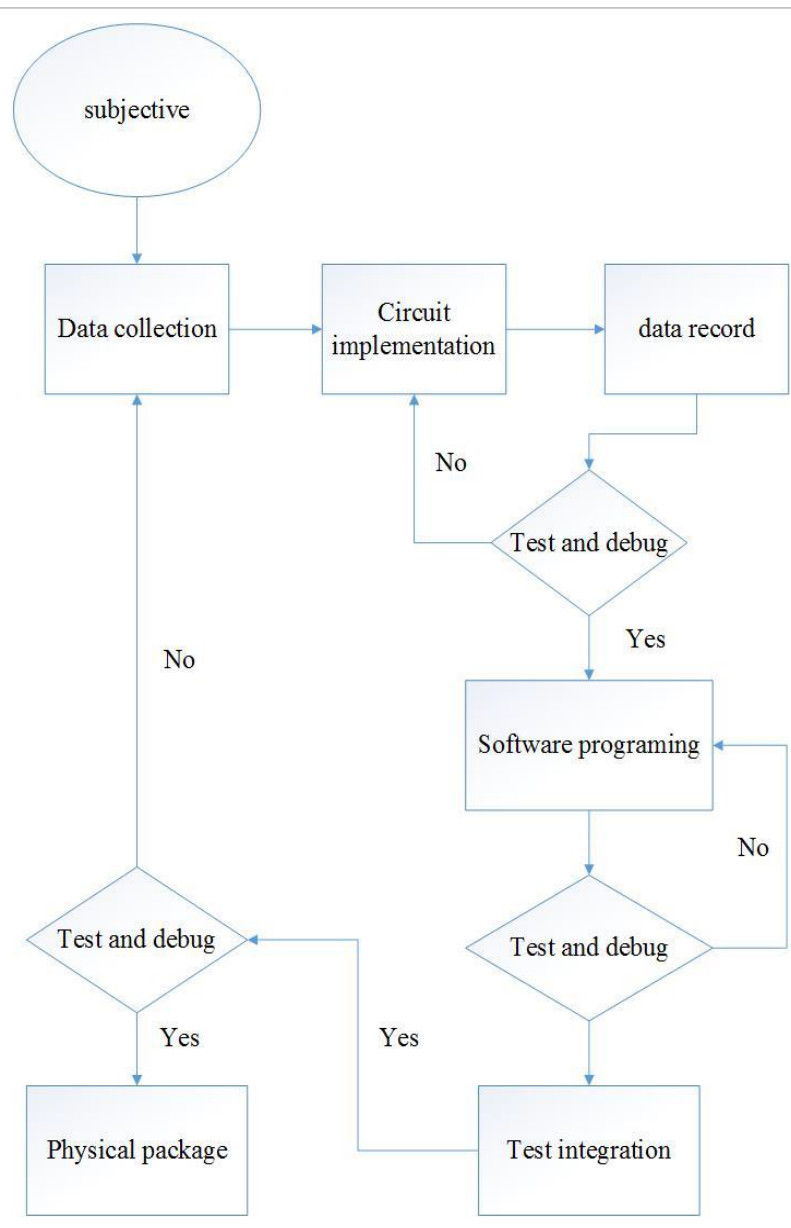

Figure 1: Flow chart of system.

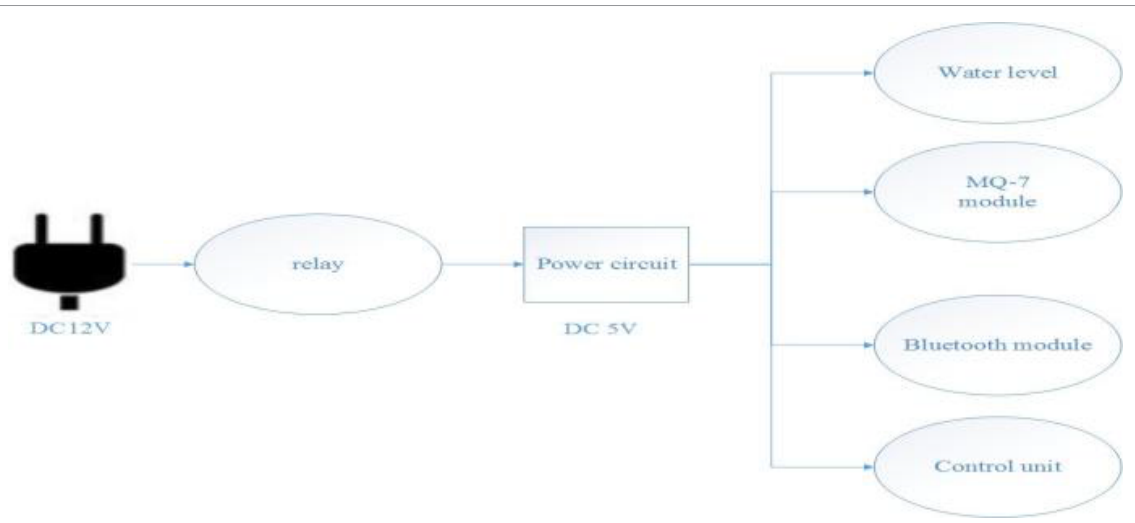

Figure 2: Diagram of entire circuit design plan.

\section{Software Structure}

\section{Arduino nano plate pin-out planning}

The chips of the Arduino Nano plate in this study use ATMEGA328P, which can be controlled by planning the required pinouts, as showed in Figure 6.

The control plate mainly uses the digital and analog pin-outs to control the sensors, which achieve integration by connecting the
Bluetooth module to a mobile phone. In emergencies, while there will be a warning alarm, the windshield-breaking device will not be immediately activated [8]. When the situation becomes more dangerous, the device will automatically break the windshield and immediately call emergency contact numbers. The overall procedure is showed in Figure 7.

\section{Sensor control}

The detection of carbon monoxide has two stages, a first stage 
Citation: Wang CS, Wang TW, Zhou MC (2017) Design of an Automatic Windshield-Breaking Device for Abnormal Situations in Moving Vehicles. J Electr Electron Syst 6: 247. doi: 10.4172/2332-0796.1000247

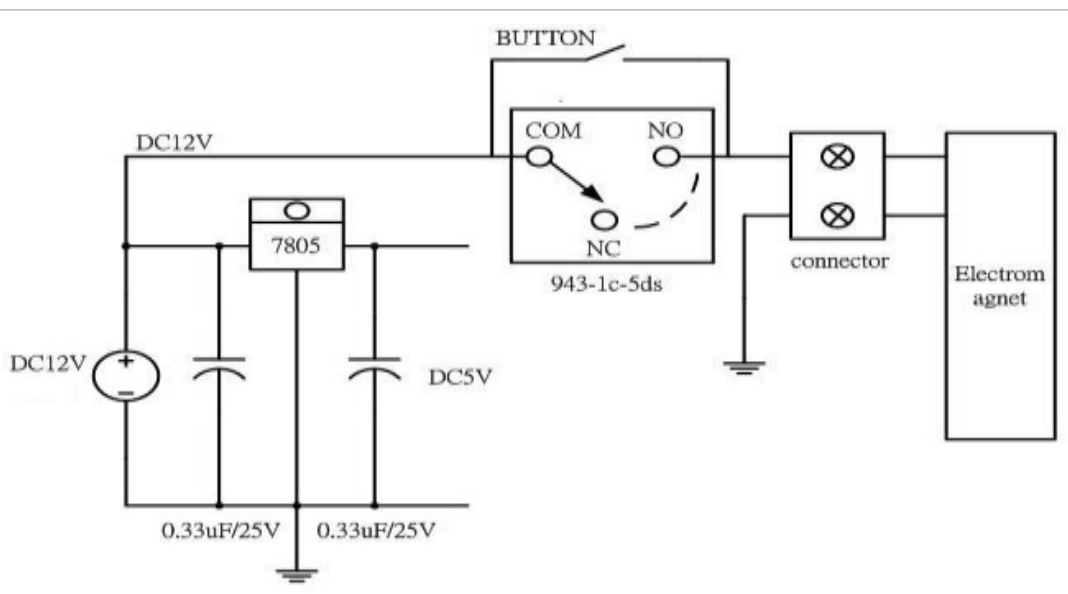

Figure 3: Electromagnet-controlled circuit.

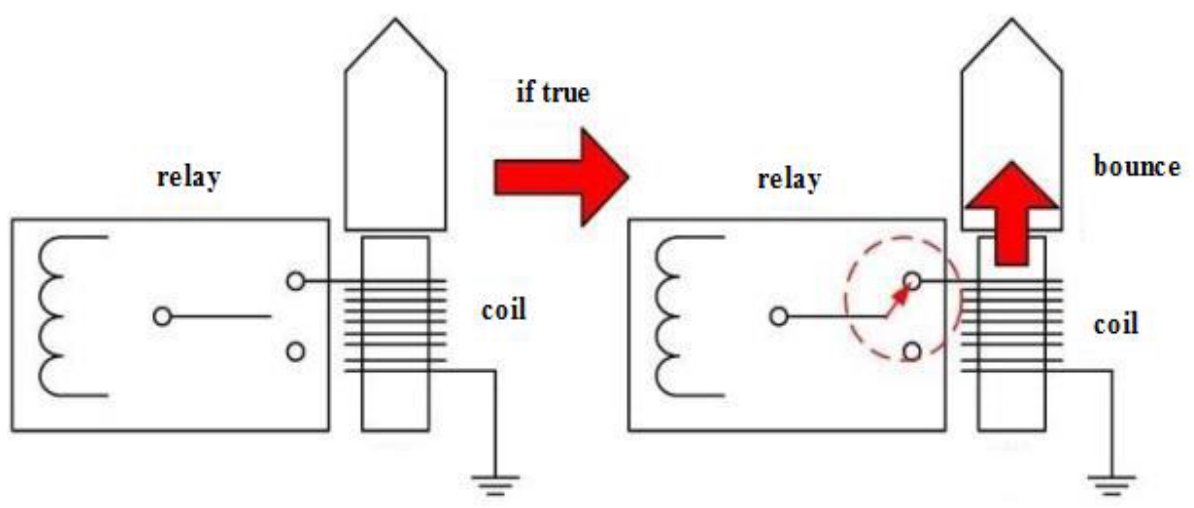

Figure 4: Diagram of the DC electromagnet system.

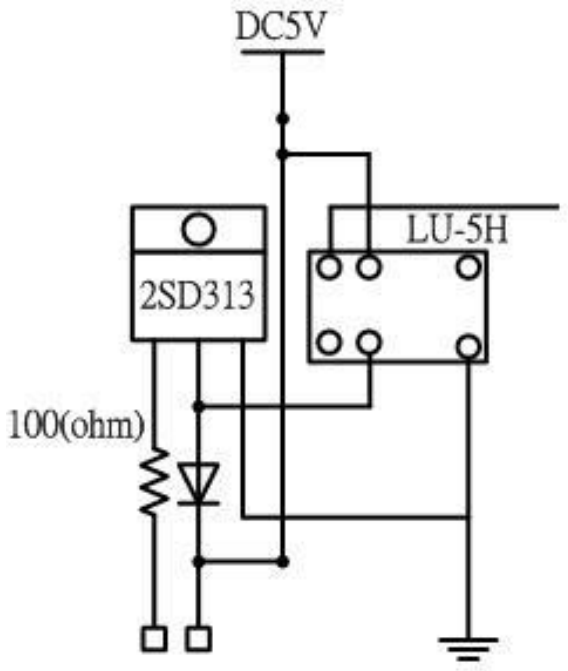

Figure 5: Water level.

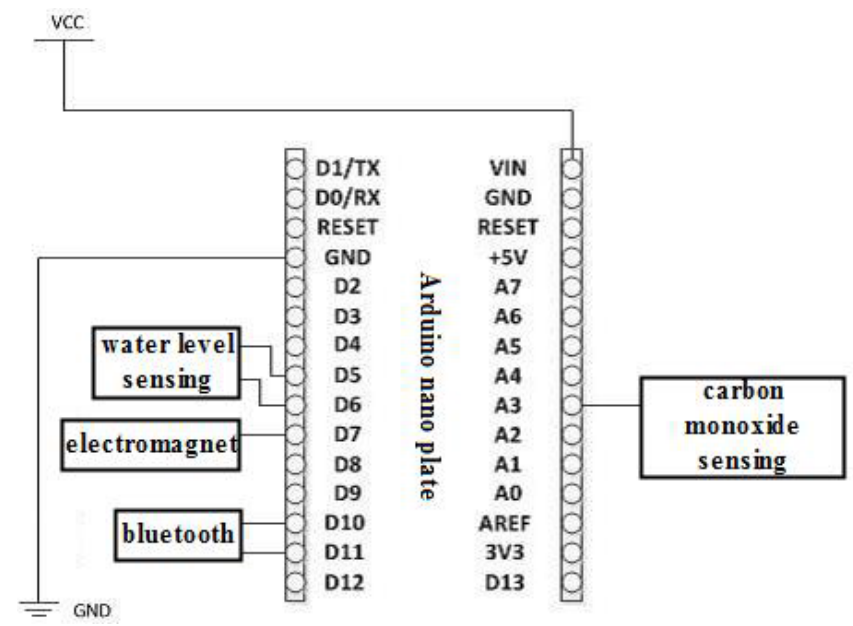

Figure 6: Diagram of pin-out planning 


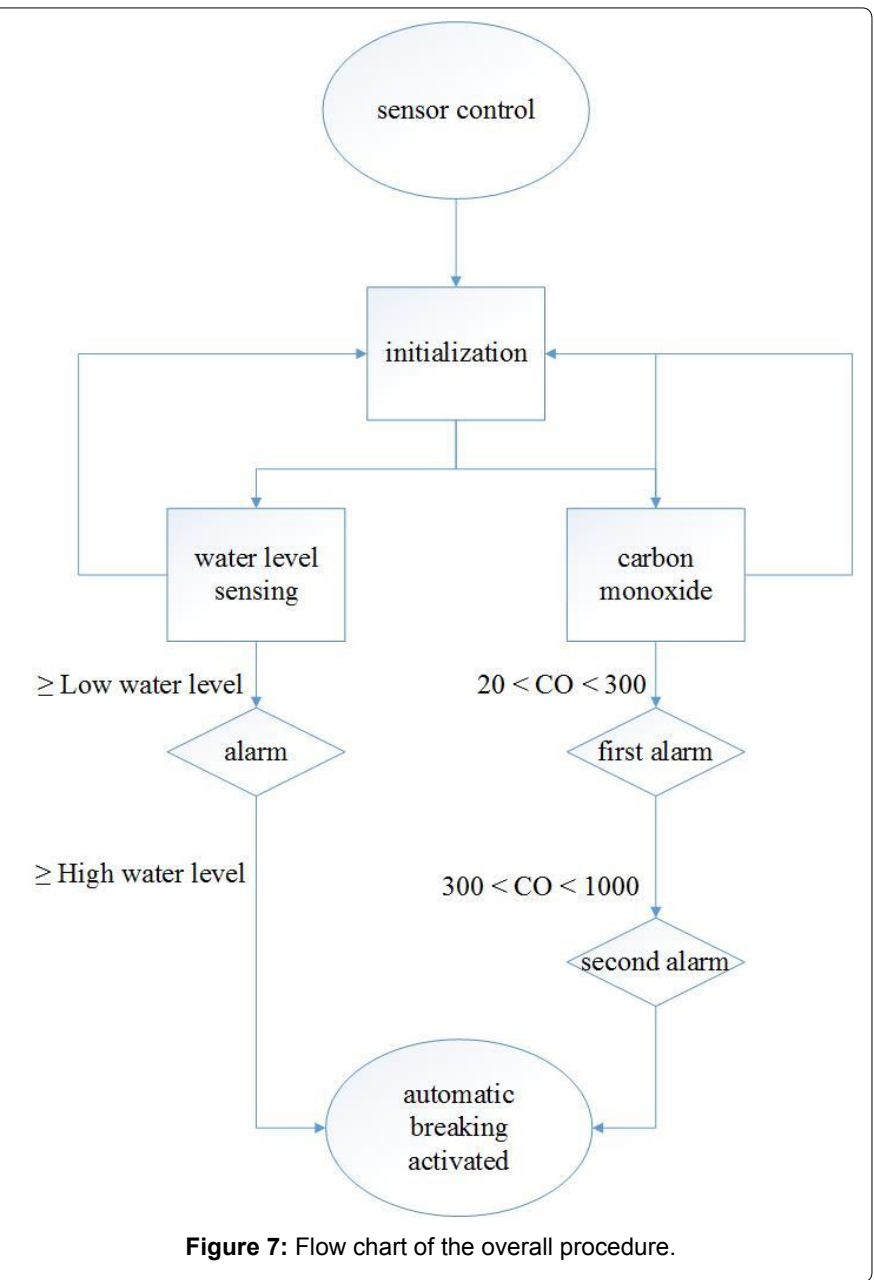

of 20-300 ppm and a second stage of 300-1000 ppm, and each has a warning tone. Only when the concentration is over $1000 \mathrm{ppm}$ will automatic breaking be activated. Water levels are divided into low and high water levels; when the water level is low, a warning will be given; when the water level is high, automatic breaking will be activated [9]. The procedure is showed in Figure 8.

\section{Android mobile phone app planning}

The mobile phone first connects with the device for the initialization actions, and when the device detects danger and breaks the windshield, it will also send a signal to trigger the automatic emergency call function. There are three main function keys on the phone interface, speed dial, contact list, and test [10]. Through the contact list button, the user can choose the emergency contact number and display it on the main screen. The speed dial button is for making emergency calls to the emergency contact, and the test button can actively send signals to the device to confirm whether the device is successfully connected. Figure 9 is the block chart of the app.

\section{Testing Effects and Functions}

The core feature of the complete system is the windshield-breaking device, which includes detecting the water level and carbon monoxide concentrations to determine the danger. The system has four functions: an automatic bounce device, a manual breaking button, a warning

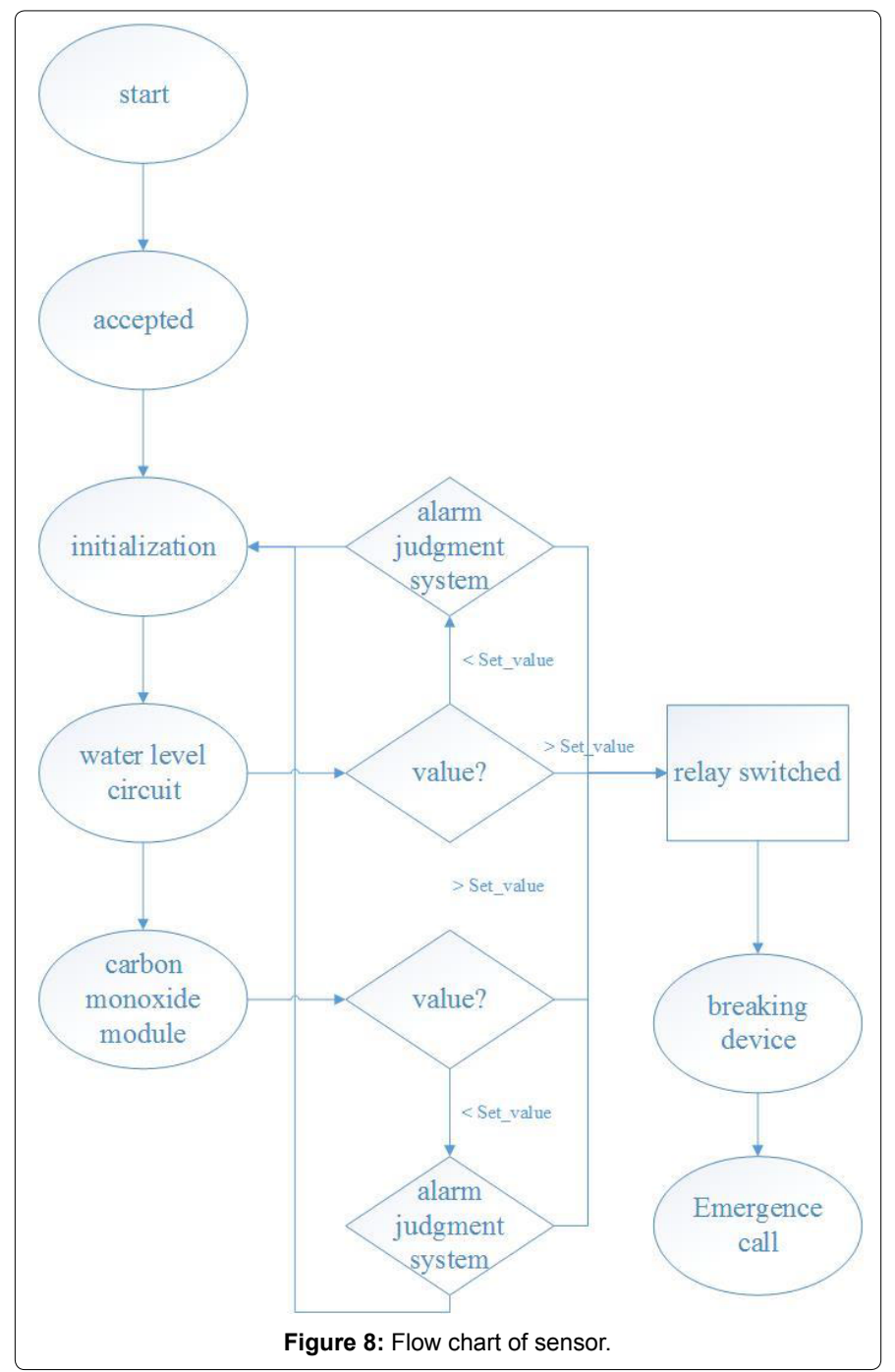

system, and emergency calling. The overall functions are shown in Figure 10.

The main procedure of the overall functions is, as follows: when the sensor detects danger, the device will automatically activate the windshield-breaking device and make emergency phone calls [11]. Its features are the abilities to break the windshield and automatically make emergency calls, which facilitate follow-up rescue actions. This is more than a normal windshield-breaking device can achieve. The functions are described, as follows:

\section{Automatic bounce}

When the sensors detect danger, the electromagnets will bounce the core via the control of the chips. In emergencies, the device can react immediately on behalf of the vehicle occupants to break the windshield.

\section{Warning system}

When the concentration of carbon monoxide is detected at 20 $300 \mathrm{ppm}$, meaning a nonfatal level, a warning sound will remind the driver of the situation for proper handling. When the concentration of carbon monoxide is detected at $300-1000 \mathrm{ppm}$, the warning sound will immediately remind the driver of the higher danger level. The water 


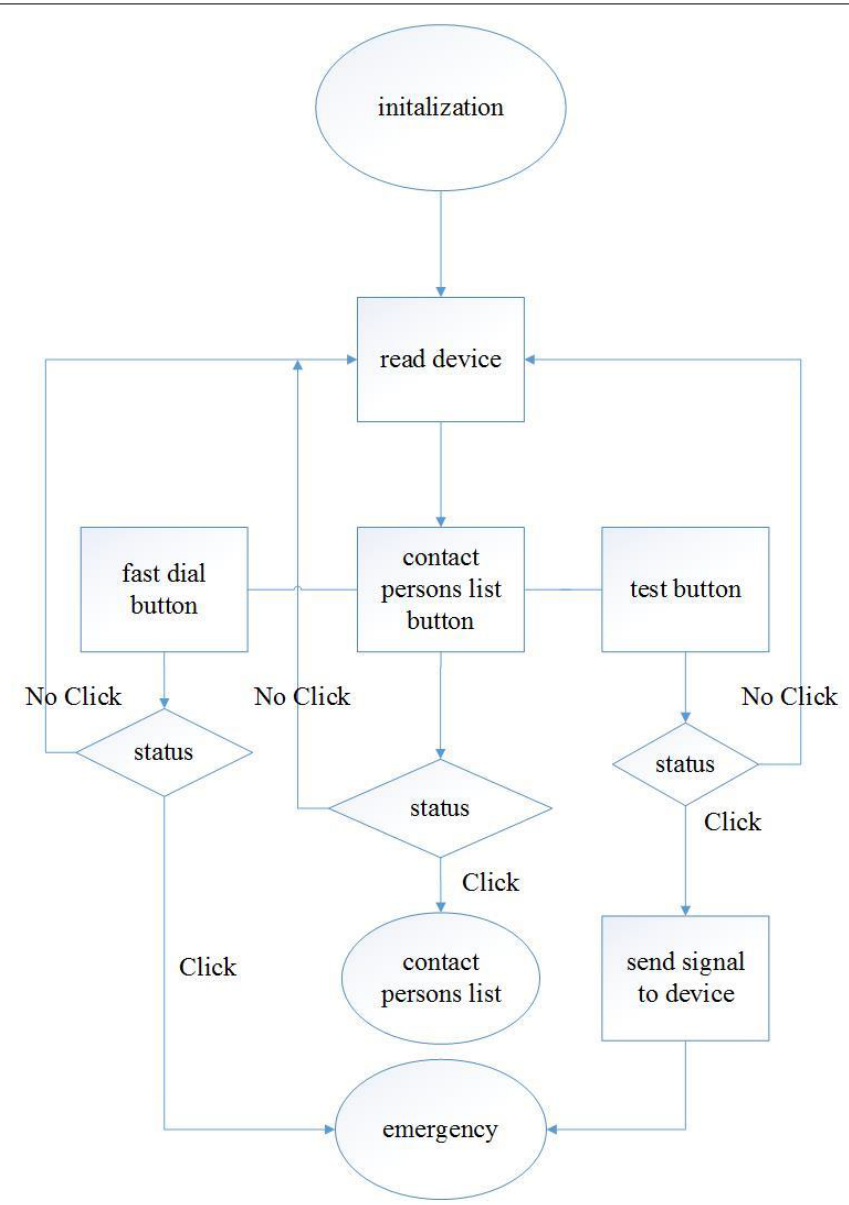

Figure 9: Block chart of android app.

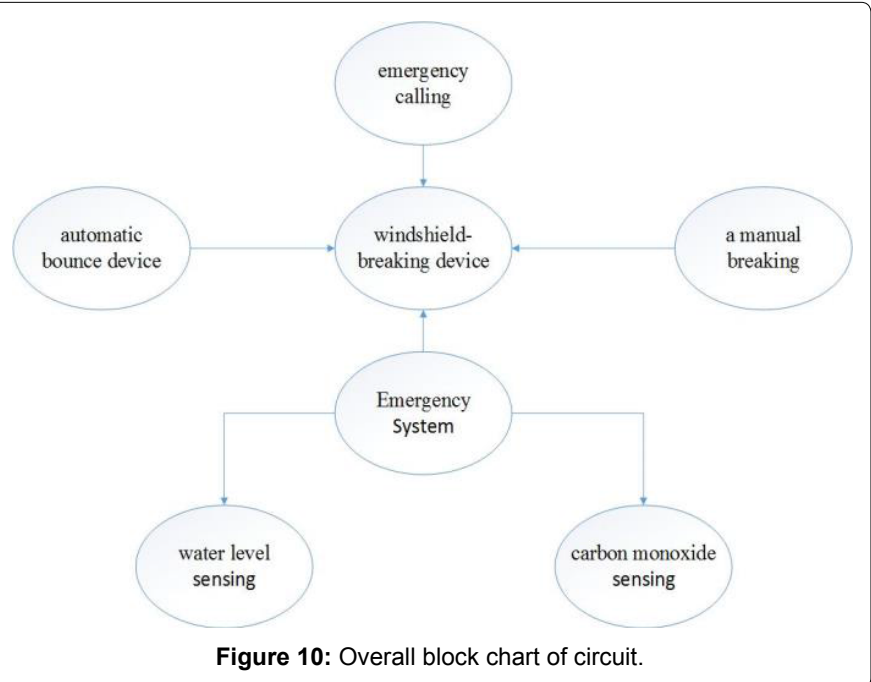

level sensor will give a warning sound to the driver when the lowest water level is reached.

\section{Manual breaking button}

During emergencies, if the driver believes their life is threatened, pressing the manual breaking button will initiate self-rescue without waiting for the automatic decision of the system [12].

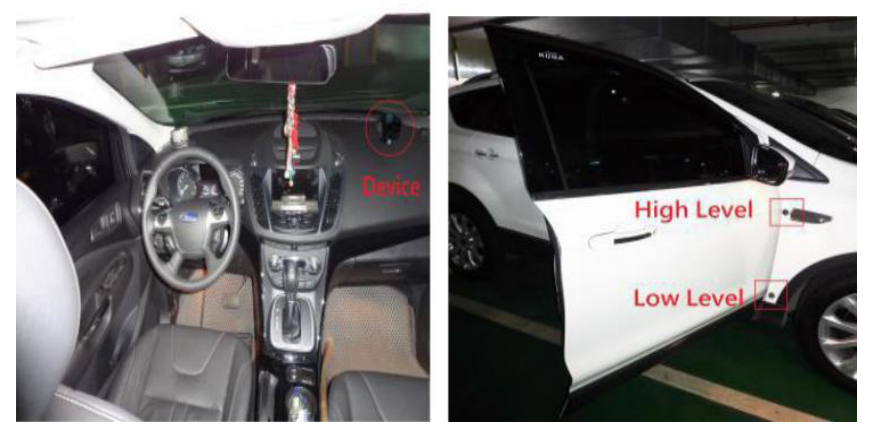

Figure 11: Diagram of device installation.

\section{Emergency calling}

After the device system automatically breaks the windshield, it will call the previously chosen emergency contact number through the Bluetooth connection with a mobile phone. Even if the driver is unconscious, rescue teams can still receive first-hand information and rush to the site. The procedures to install this systematic device in a vehicle are shown in Figure 11.

\section{Conclusion}

This study conducts the integrated application of Arduino control and Android mobile phones, and focuses its research direction on a windshield-breaking device, which aims to improve the existing products on the market that are not able to automatically break the windshield, detect dangers, or immediately provide the best chance of rescue during emergencies. The completion of this study shortens the escape time and increases the chance of escape. The final goal of this study is to cooperate with car manufacturers to promote this device as a standard or optional accessory, thus, maximizing its effects to ensure the safety of the occupants of the vehicle.

\section{References}

1. Ho YH, Yap DFW, Rahman MFA, Kok SL (1982) Bandwidth widening strategies for piezoelectric based energy harvesting from ambient vibration sources. IEEE International Conference on Computer Applications and Industrial Electronics.

2. Knysh SI, Chen EH, Durkin GA (2010) True Limits to Precision via Unique Quantum Probe. Quantum Physics.

3. Tsai YH, Chang MJ, Chen WZ, Wang JB, Lai JC (2011) Muti-functional escape window. Department of Electrical Engineering, Technology and Science Institute of Northern Taiwan.

4. Chen C, Liu Z, Zhang Y, Chen CLP, Xie SL (2017) Asymptotic Fuzzy Tracking Control for a Class of Stochastic Strict-Feedback Systems. IEEE Transactions on Fuzzy Systems 25: 556-568.

5. Chang JC, Hsiao FZ, Huang JC, Kao SP, Li HC, et al. (1993) Finite Element Analysis on Helium Discharge from Superconducting RF in the Storage Ring Tunnel. JACoW.

6. Huang WF, Hung KJ, Huang DC, Huang CC, Tseng PK (2000) Positron spectroscopy of base-adsorbed Y-zeolite. Phys Lett 266: 430-434.

7. Shi WM (2015) Android app design textbook. Flag

8. Kumar S (2008) Design and fabrication of micro-controller based $120 \mathrm{~Hz}$ earth tester. Electrical and Instrumentation Engineering, Thapar University.

9. Zhao YJ (2015) Arduino interactive design. Flag

10. Chang HS (2013) Computer auxiliary circuit design by using CADSTAR Taiwan Tech Press, Taiwan.

11. https://www.arduino.cc/

12. http://www.car 